\title{
Unified access in licensed and unlicensed bands in LTE-A Pro and 5 G
}

\author{
BOON LOONG NG, HONGBO SI, ARIS PAPASAKELLARIOU AND JIANZHONG CHARLIE ZHANG
}

\begin{abstract}
Spectrum scarcity has driven enhancements of Long-Term Evolution (LTE) in utilizing unlicensed bands in conjunction with licensed bands for delivering mobile data, resulting in the introduction of LTE unlicensed technologies such as Rel-13 LTELicensed-Assisted Access (LAA), Rel-14 LTE-Enhanced Licensed-Assisted Access (eLAA), and LTE-Unlicensed (LTE-U). The next-generation radio access technology, ${ }_{5} G$ New Radio(NR), faces greater technical challenge due to the need to support frequency bands covering various spectrum licensing regimes and a wide range of frequencies (up to $100 \mathrm{GHz}$ ) with very different signal propagation characteristics. This paper presents an overview of LAA and eLAA technical features and ${ }_{5} G$ NR design considerations to achieve a unified access in licensed and unlicensed bands.
\end{abstract}

Keywords: 5 G, LTE-A, Unlicensed band

Received 2 December 2016; Revised 5 June 2017

\section{INTRDDUCTION}

To meet the demand of mobile traffic growth, there has been increasing interest by cellular operators to utilize unlicensed spectrum to complement Long-Term Evolution (LTE) systems operating in licensed spectrum. Licensed spectrum has traditionally afforded operators mechanisms for guaranteeing Quality of Service and seamless mobility, while unlicensed spectrum operation leads to unique challenges for cellular networks due to the need for co-existence with $\mathrm{Wi}-\mathrm{Fi}$ that has been the dominant radio access technology (RAT) for these bands until now.

In 2013, the 3rd Generation Partnership Project (3GPP) approved a new study item on Licensed-Assisted Access (LAA) to investigate the feasibility of extending LTE operation to unlicensed bands [1]. This was extended to a work item in 2014 [2] as part of Rel-13 LTE-Advanced Pro (LTEA Pro) to standardize a single global solution framework ensuring LAA can meet all regulatory requirements of different regions, such as those from the Federal Communications Commission (FCC) for the USA [3] and the European Telecommunications Standards Institute (ETSI) for the EU [4], and co-exist with other operators or other RATs, such as Wi-Fi, operating on the same band. LAA only supports downlink transmissions on unlicensed bands, where respective carriers are configured as secondary carriers as part of carrier aggregation with at least one carrier on a

Samsung Research America, Richardson, Texas, USA

Corresponding author:

J.C. Zhang

Email: jianzhong.z@samsung.com licensed band. The LTE-U forum, a parallel industry forum formed in 2014, also specified support of LTE in unlicensed bands [5]. Since then, a second ${ }_{3} \mathrm{GPP}$ work item, called enhanced LAA (eLAA) [6], was completed in 2016 as a part of Rel-14 LTE-A Pro. eLAA continues from LAA and additionally enables support of uplink transmissions on unlicensed bands [7-9]. From the beginning of the $3 \mathrm{GPP}$ study item on LAA until the completion of two work items on LAA and eLAA, extensive evaluations were conducted in 3 GPP to determine how LAA/eLAA can co-exist with existing Wi-Fi deployments in a fair manner for a variety of deployment scenarios (see [10] for comprehensive evaluation results). Co-existence among LAA/eLAA networks deployed by different operators is also an important design consideration since coordination and synchronization between nodes of different operators cannot be typically assumed.

As spectrum availability for initial ${ }_{5} \mathrm{G}$ New Radio (NR) can be scarce in at least some world regions and as unlicensed bands practically represent global bands, support of unlicensed band operations continues to be an important requirement also for $5 \mathrm{G} \mathrm{NR}$ [11]. Completion of specifications is expected by the end of 2019. The ${ }_{5} \mathrm{G} \mathrm{NR}$ aims to support a very wide range of carrier frequencies up to $100 \mathrm{GHz}$. Hence, unlike LAA/eLAA that only provide support for the $5 \mathrm{GHz}$ unlicensed band (although future extension to include additional frequency bands is possible), $5 \mathrm{G} \mathrm{NR}$ is required to cover a wide range of unlicensed and shared licensed frequency bands, where regulatory and inter-RAT co-existence requirements may differ for each band. This includes the $3.5 \mathrm{GHz}$ band that has been designated as a shared band in the USA as well as the 5 and $60 \mathrm{GHz}$ bands 
that are unlicensed bands. Moreover, system design needs to consider the vastly different wireless channel characteristics for the lower carrier frequency bands such as at $<6 \mathrm{GHz}$ and higher carrier frequency bands such as at $60 \mathrm{GHz}$. As a result of the inclusiveness of $5 \mathrm{G} \mathrm{NR}$, a unified access framework for licensed, unlicensed, and shared licensed bands is a highly desirable and challenging system design goal for $5 \mathrm{G}$ NR. Nevertheless, $5 \mathrm{G}$ NR operations are expected to vary in details for bands with different licensing regimes due to the different regulatory and co-existence requirements.

This paper is organized as follows. Section II provides an overview of Rel-13 LAA and Rel-14 eLAA key features. Section III discusses $5 \mathrm{G}$ NR design considerations for unlicensed and shared access spectrum. Finally, conclusions are presented in Section IV.

\section{UNLICENSED ACLESS FDR LTE-A PRD}

\section{A) Rel-13 LTE-LAA}

LAA operates on the $5 \mathrm{GHz}$ unlicensed spectrum consists of three subbands: 5.17-5.33, 5.49-5.725, and 5.725-5.835 GHz. Each subband can be further divided into non-overlapped $20 \mathrm{MHz}$ channels according to IEEE 802.11ac [12] channelization. The ${ }_{5} \mathrm{GHz}$ unlicensed spectrum is of particular importance due to its global availability as shown in Fig. 1.

\section{1) Channel access}

Listen-before-talk (LBT) is adopted as the fundamental coexistence mechanism for LAA, whereby a radio transmitter is required to apply a clear channel assessment (CCA) check prior to transmission. CCA involves at least energy detection (ED) over a time duration with a certain threshold (ED threshold) to determine if a channel is occupied or is clear. If the channel is occupied, random back-off within a contention window applies, so that there is a minimum time duration where the channel is clear before the transmitter can transmit. In order to protect Wi-Fi ACK transmissions, a defer period (e.g. $43 \mu$ s for best effort traffic) is applied after each busy CCA slot before resuming back-off. After the transmitter has gained access to the channel, the transmitter is only allowed to transmit for a limited duration referred to as the maximum channel occupancy time (MCOT). To provide differentiation to channel access priorities based on the type of traffic served (e.g. VoIP, video, best effort, or background), four LBT priority classes are defined with different contention window sizes (CWS) and MCOT. An illustrative flowchart of the LAA LBT procedure is given in Fig. 2. Table 1 summarizes the MCOT and CWS for the four priority classes.

ED Threshold. Since the ED threshold determines whether or not a channel is occupied, it is one of the most critical parameters concerning co-existence. The ${ }_{3} \mathrm{GPP}$ has adopted a scenario-dependent ED threshold adaptation rule. In the presence of other technology, such as Wi-Fi sharing the spectrum, the ED threshold $(T H)$ is given by

$$
T H=\max \left\{\begin{array}{c}
-72 \mathrm{dBm}(20 \mathrm{MHz}), \\
\min \left\{T_{\max }, T_{\max }-T_{A}+\left(P_{H}-P_{T X}\right)\right\}
\end{array}\right\},
$$

where $T_{\text {max }}=-75(\mathrm{dBm} / \mathrm{MHz})+10 \cdot \log _{10}(B), B$ is the single channel/carrier bandwidth in $\mathrm{MHz}, T_{A}$ is an adjustment parameter that depends on the transmission type (10 dB for data and $5 \mathrm{~dB}$ for Discovery Reference Signal (DRS)), $P_{H}=23 \mathrm{dBm}$, and $P_{T X}$ is the maximum base station (eNB) output power in $\mathrm{dBm}$ set for the carrier. For example, at $P_{T X}$ of $23 \mathrm{dBm}$ (typical for an indoor deployment scenario), the ED threshold for data transmission over a $20 \mathrm{MHz}$ channel is $-72 \mathrm{dBm}$, whereas the ED threshold for DRS transmission is $-67 \mathrm{dBm}$. Nevertheless, the ED threshold is allowed to increase in case of wider channel bandwidth (while keeping the total transmission power constant) or in case of a lower total transmission power.

In absence of other co-existing technology, the ED threshold can be increased to $T_{\max }+10 \mathrm{~dB}$ (subject to regional regulatory requirements) that can result to a threshold as high as $-52 \mathrm{dBm}$ for a $20 \mathrm{MHz}$ channel. The high ED threshold is beneficial for facilitating a frequency reuse factor of one among neighboring LAA cells.

CWS adaptation. Similar to CWS adaptation design utilized by Wi-Fi, LAA supports dynamic CWS adaptation based on the hybrid automatic repeat request - acknowledgement (HARQ-ACK) feedback corresponding to the transport blocks sent over the LAA carrier. In particular, the CWS is increased if at least $80 \%$ of the HARQ-ACK values corresponding to transmissions in the first subframe of a downlink (DL) burst are NACK/DTX; otherwise, the CWS is reset to the minimum value. The detailed minimum and maximum CWS and the set of allowed CWS values for different priority classes were designed to match those of Wi-Fi

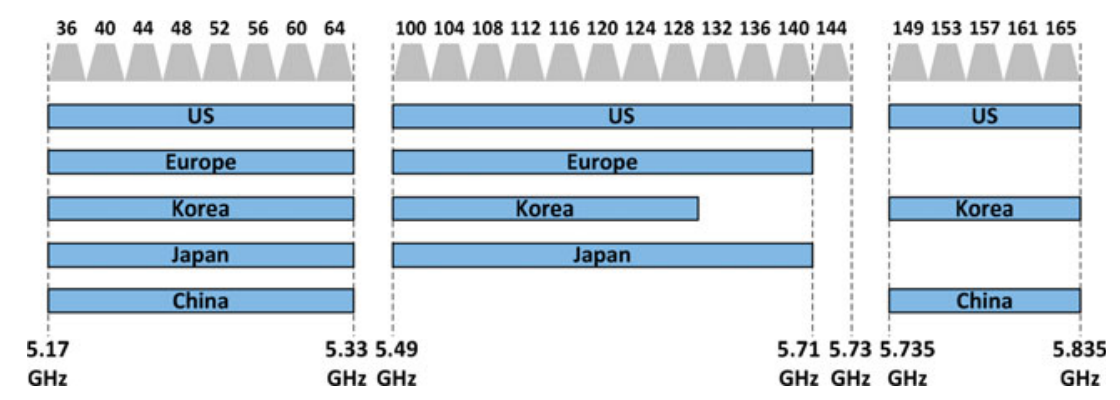

Fig. 1. Worldwide $5 \mathrm{GHz}$ unlicensed spectrum allocation. 


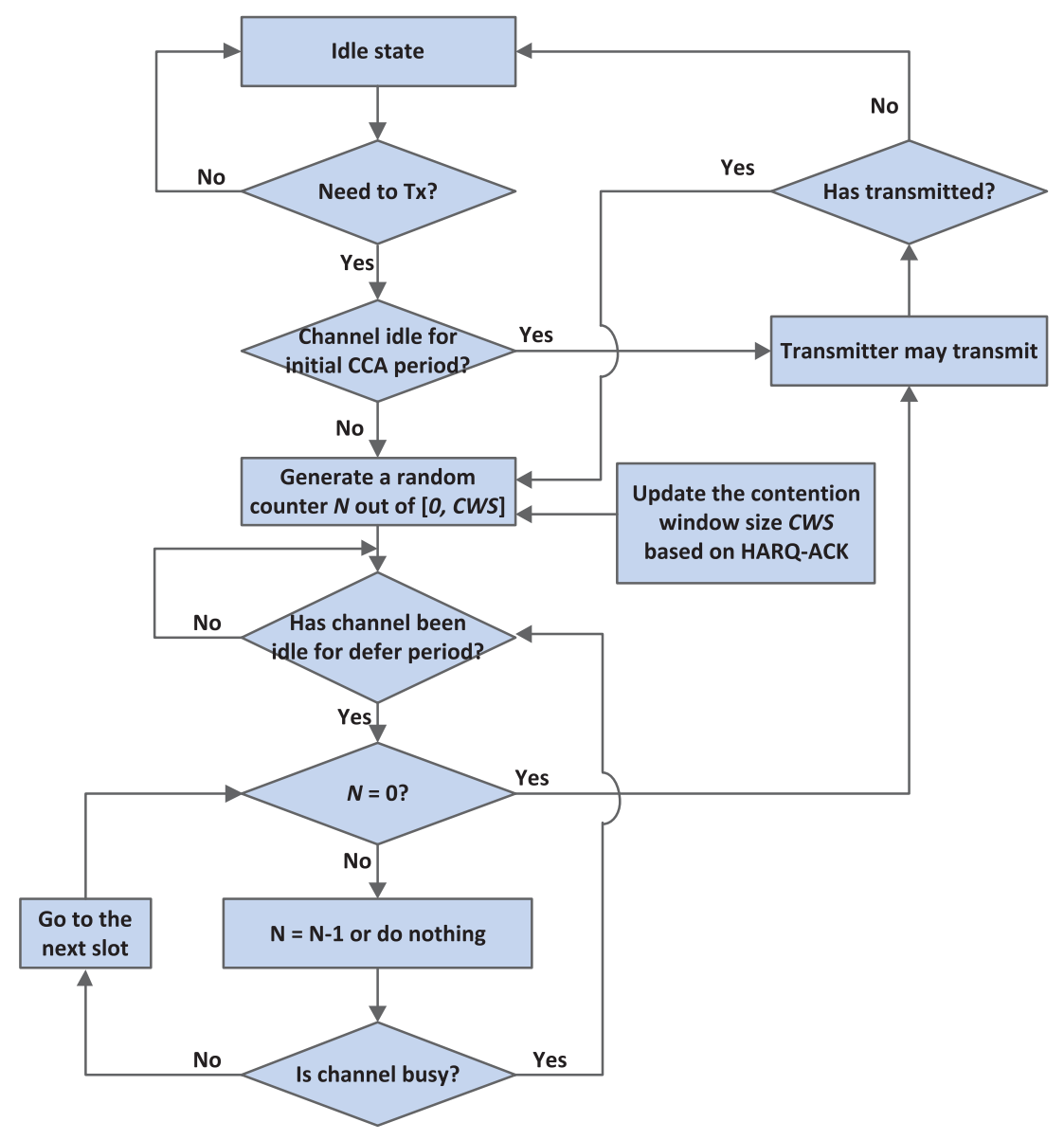

Fig. 2. Flowchart of LAA DL LBT procedure.

Table 1. Channel access priority class for downlink.

\begin{tabular}{lll}
\hline Priority class & MCOT $(\mathrm{ms})$ & CWS \\
\hline 1 & 2 & $\{3,7\}$ \\
2 & 3 & $\{7,15\}$ \\
3 & 8 or 10 & $\{15,31,63\}$ \\
4 & 8 or 10 & $\{15,31,63,127,255,511,1023\}$ \\
\hline
\end{tabular}

Note 1: Defer period is different for different priority class. Details are omitted here for brevity.

Note 2: MCOT of $8 \mathrm{~ms}$ for priority classes 3 and 4 is used in the presence of other technology; MCOT of $10 \mathrm{~ms}$ is used otherwise.

(see Table 1). For example, for LBT priority class 3 (best effort traffic), the set of allowed CWS values is $\{15,31,63\}$ and it effectively realizes exponential back-off.

\section{2) LAA DISCOVERY REFERENCE SIGNAL}

The DRS is a set of signals that includes the Primary Synchronization Signal, the Secondary Synchronization Signal, the Cell-specific Reference Signal, and the Channel State Information Reference Signal (if configured). DRS transmission is utilized in LAA for cell detection, synchronization, and radio resource management (RRM) measurement. Similar to the Rel-12 DRS, LAA DRS can be transmitted within a periodically occurring time window called the
DRS measurement timing configuration (DMTC) occasion that has a duration of $6 \mathrm{~ms}$ and a configurable period of 40/80/16o ms. However, to reduce a collision probability, the transmission of DRS is also subject to LBT. Specifically, DRS can be transmitted following a single idle observation interval of at least $25 \mu \mathrm{s}$. To compensate for potential DRS transmission blocking due to LBT and increase the probability of successful DRS transmission, the network is allowed to attempt DRS transmission in any subframe within the DMTC occasion. This implies that LAA DRS transmission is not strictly periodic as shown in Fig. 3.

\section{3) LAA FRAME STRUCTURE}

LAA has introduced the concept of a "partial subframe" whereby a DL transmission may start at the first or second slot boundaries of a subframe. Limiting the starting positions to slot boundaries helps reduce the complexity of eNB transmission preparation and user equipment (UE) blind detection of the starting positions. DL transmission also may not end at the subframe boundary. The existing Downlink Pilot Time Slot (DwPTS) structure is reused where DwPTS is the DL portion of the special subframe of the LTE Frame Structure Type 2 (used for time division duplex operation). To simplify UE implementation for subframe processing, common control signaling from a LAA SCell is used to indicate the number of orthogonal frequency 


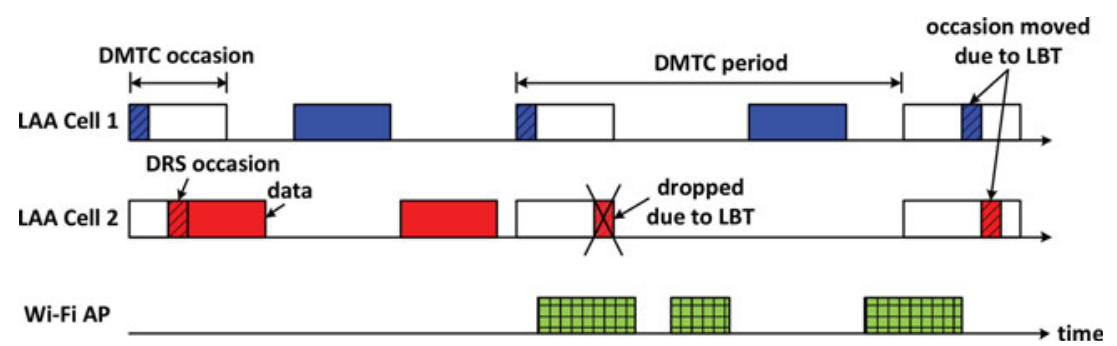

Fig. 3. LAA DRS transmission.

division modulation (OFDM) symbols of a current and a next subframe for DL transmission.

\section{4) RAdio Resource management}

Channel selection for LAA is very important for coexistence with other RATs such as Wi-Fi. For example, LAA should try to avoid frequencies that are more congested with Wi-Fi [access points/station (APs/STAs)] and RRM measurements are critical for this purpose. In legacy LTE operation, Reference Signal Received Power (RSRP), Received Signal Strength Indicator (RSSI), and Reference Signal Received Quality (RSRQ) are specified and only RSRP and RSRQ can be reported to an eNB by a UE. RSSI can serve as a metric for the interference strength on a carrier, and it is possible to infer RSSI from RSRP and RSRQ reports. However, if the DRS is not transmitted by the eNB on a carrier, for example due to LBT, RSRP and RSRQ reports would not be available. As a result, RSSI measurement reports along with time information about when the measurements were made by a UE are useful for hidden node detection at an LAA eNB.

The absolute RSSI level observed by a UE as well as statistics of RSSI distribution during a measurement period are useful to provide a more complete picture of the load on a carrier and assist in hidden node detection by correlating measurements made by one or more UEs with those made by the eNB. As a result, LAA has introduced new measurements of average RSSI and channel occupancy (percentage of time that RSSI was observed above a configured threshold) for RRM reports. To this end, a RSSI measurement timing configuration (RMTC) can be configured to indicate a measurement duration (e.g. 1-5 ms) and period between measurements (e.g. $\{40,80,160,320,640\} \mathrm{ms})$.

Figure 4 illustrates an exemplary RMTC configuration with an averaging granularity of one OFDM symbol, measurement duration of 70 symbols (e.g. $5 \mathrm{~ms}$ ) with a periodicity of $40 \mathrm{~ms}$, and a total measurement period comprising three measurement durations (e.g. $120 \mathrm{~ms}$ ), and resulting average RSSI and channel occupancy measurement reports.

\section{B) Rel-14 LTE-eLAA}

\section{1) Channel access}

LBT is also mandatory for uplink channel access. Rel-14 eLAA supports two types of uplink channel access procedures. Type 1 uplink channel access procedure is analogous to the one for downlink channel access in Rel-13 LAA where a series of slots for CCA have to be sensed as clear on a channel before a UE can transmit on the channel. As for the downlink, the number of slots is generated from a CWS that is adaptively adjusted by a UE. Four types of priority classes are also supported in the uplink; however, particular values for MCOT and CWS are different from the ones in downlink (see Table 2). Type 2 uplink channel access procedure is analogous to the one for DRS transmission where a UE performs CCA over only a short period. The duration of the period is fixed to be at least $25 \mu \mathrm{s}$.

For transmission of a Physical Uplink Shared Channel (PUSCH), an eNB indicates to a UE the type of channel

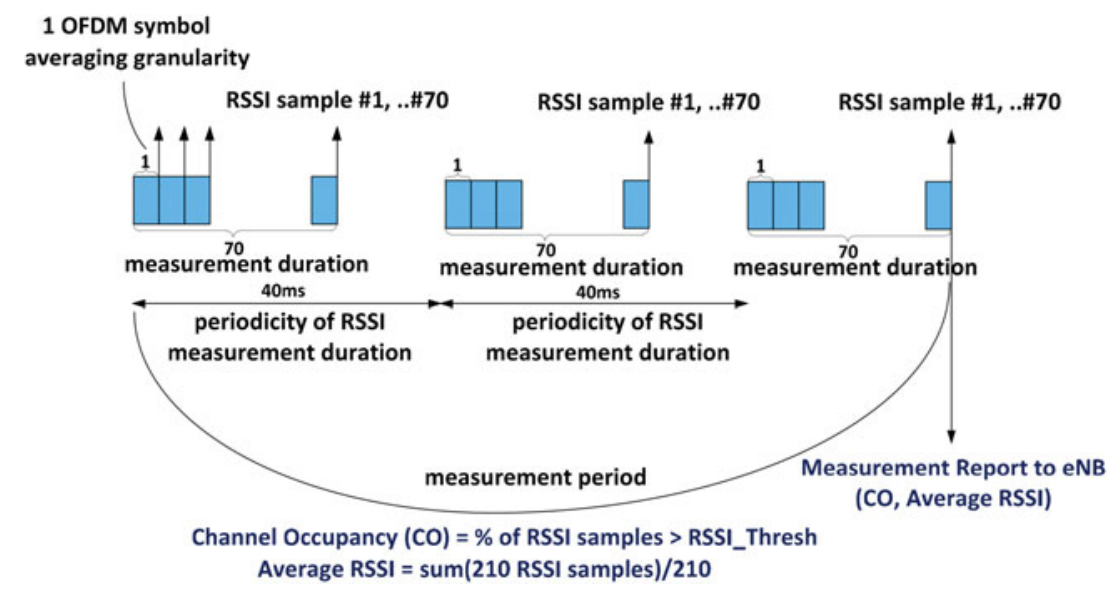

Fig. 4. Example of RMTC configuration with averaging granularity of one OFDM symbol, measurement duration of 70 symbols (e.g. 5 ms) with a periodicity of $40 \mathrm{~ms}$, and a total measurement period comprising three measurement durations (e.g. $120 \mathrm{~ms}$ ). 
Table 2. Channel access priority class for uplink.

\begin{tabular}{lll}
\hline Priority class & MCOT $(\mathrm{ms})$ & CWS \\
\hline 1 & 2 & $\{3,7\}$ \\
2 & 3 & $\{7,15\}$ \\
3 & 6 or 10 & $\{15,31,63,127,255,511,1023\}$ \\
4 & 6 or 10 & $\{15,31,63,127,255,511,1023\}$ \\
\hline
\end{tabular}

Note 1: The MCOT in uplink does not include gaps of durations $>25 \mu$ s that occur between transmissions.

access procedure through an uplink grant scheduling the PUSCH transmission. In general, type 1 uplink channel access procedure is utilized to initialize a MCOT containing PUSCH transmission, while type 2 uplink channel access procedure is utilized within the MCOT for resuming a suspended transmission or for changing the transmission direction from downlink to uplink. For a transmission of a Sounding Reference Signal without a PUSCH from a UE, the UE shall always utilize type 1 uplink channel access procedure with the highest priority class.

The maximum ED threshold requirement for a UE is aligned with the requirement for an eNB as specified in Rel13 LAA unless the UE is configured by higher layers to use a separate maximum ED threshold.

After performing type 1 channel access procedure, a UE determines its own reference subframe as the first subframe within the most recent burst for adjusting the CWS for type 1 uplink channel access procedure. The CWS is increased to the next available value when the transmission corresponding to the reference subframe fails; otherwise, the CWS is reset to the minimum value.

\section{2) PUSCH DESIGN}

To meet regulation requirements in respective world regions for spectral occupancy of an uplink transmission on unlicensed spectrum while allowing full UE transmit power utilization, the basic resource allocation unit for a PUSCH transmission is one interlace that includes 10 resource blocks (RB) that are spaced equally in frequency. An uplink transmission can be over one or more interlaces. A total number of RBs should be a multiple of 2, 3, and 5 in order to minimize a complexity of a discrete fourier transform filter.

Due to the time uncertainty associated with channel access, Rel-14 eLAA supports flexible timing between the subframe of an uplink grant transmission and the subframe of an associated PUSCH transmission with a minimum latency of $4 \mathrm{~ms}$ in order to avoid increasing UE processing complexity relative to legacy LTE operation. Rel-14 eLAA also supports multi-subframe scheduling, where an uplink grant can schedule PUSCH transmissions in a number of multiple consecutive subframes ranging from two to four subframes. Specifically, Rel-14 eLAA supports dynamic control information (DCI) formats $\mathrm{OA}$ and $4 \mathrm{~A}$ for single subframe scheduling and DCI formats $\mathrm{OB}$ and $4 \mathrm{~B}$ for multi-subframe scheduling for single-layer and multi-layer PUSCH transmissions, respectively. In order to enable faster PUSCH transmissions without increasing UE complexity,
Rel-14 eLAA also supports a two-stage uplink scheduling where a first uplink grant provides the scheduling information for a UE to prepare a PUSCH transmission and a second grant transmitted in a later subframe, also referred to as triggering grant, triggers the $\mathrm{PUSCH}$ transmission.

\section{UNLICENSED AND SHARED ACCESS FOR $5 G$ NR}

At least three unlicensed or shared spectrum bands are considered for 5 G NR: $3.5,5$, and $60 \mathrm{GHz}$. Since 3 GPP Rel-13 LAA and Rel-14 eLAA have already established downlink and uplink operations on the $5 \mathrm{GHz}$ unlicensed spectrum, with consideration to fair co-existence with existing RATs, these schemes can offer a starting point for the design of $5 \mathrm{G} N R$ on this unlicensed spectrum. For the remaining two spectrums that allow operation in a license-free or licenseshared manner, the detailed operation of $5 \mathrm{G}$ NR may not be the same due to different features, regulation requirements, and co-existing RATs in the different bands. The following subsections provide an overview of the spectrum allocations, the regulatory requirements, and the channel access characteristics for 60 and $3.5 \mathrm{GHz}$, followed by a brief discussion on the $5 \mathrm{G}$ NR unifying framework for licensed and unlicensed bands.

\section{A) The $60 \mathrm{GHz}$ unlicensed spectrum}

The $60 \mathrm{GHz}$ unlicensed spectrum refers to the licensefree millimeter-wave bands ranging from 57 to $66 \mathrm{GHz}$. These bands have unique characteristics that make them significantly different from the traditional $5 \mathrm{GHz}$ unlicensed spectrum.

Figure 5 illustrates the unlicensed spectrum allocation for major geographic areas worldwide and also includes the IEEE 802.11ad [12] channelization. Overall, the $60 \mathrm{GHz}$ unlicensed spectrum supports up to four non-overlapping channels, where each channel has a bandwidth of $2.16 \mathrm{GHz}$, and enables four channels in Europe; three channels in the USA, Canada, Korea, and Japan; and two channels in China. Requirements and regulations on the equivalent isotropically radiated power (EIRP) for $60 \mathrm{GHz}$ vary by geographic areas and are different from the ones at $5 \mathrm{GHz}$. For example, the requirement for peak EIRP is $43 \mathrm{dBm}$ in the USA and $4 \mathrm{O} \mathrm{dBm}$ in Europe where the maximum power spectral density is also required to be $13 \mathrm{dBm} / \mathrm{MHz}$.

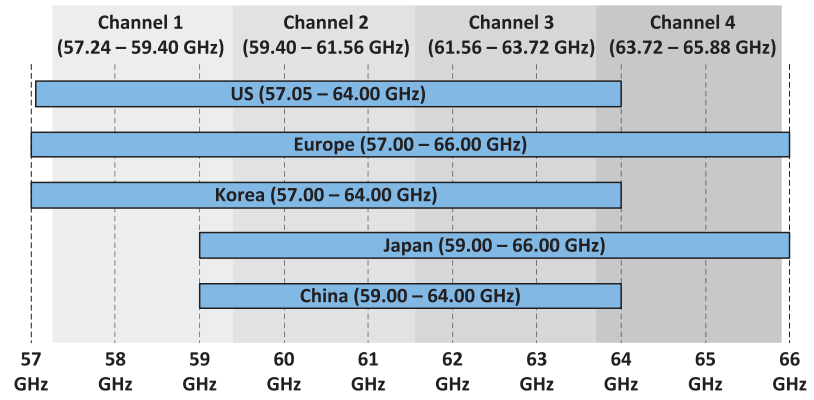

Fig. 5. Worldwide $60 \mathrm{GHz}$ unlicensed spectrum allocation. 
The primary challenge with millimeter-wave operation on $60 \mathrm{GHz}$ is the much larger free space pass loss compared with operation on $5 \mathrm{GHz}$. The power loss over $1 \mathrm{~m}$ at $60 \mathrm{GHz}$ can be as large as $68 \mathrm{~dB}$, and this is $21.6 \mathrm{~dB}$ worse than at $5 \mathrm{GHz}$. The obstruction loss, even from people or foliage, is also much higher at $60 \mathrm{GHz}$ than it is at $5 \mathrm{GHz}$. However, the high propagation loss at $60 \mathrm{GHz}$ can be mitigated by increasing the antenna gain as the small geometries required at $60 \mathrm{GHz}$ enable the use of physically small and high-gain directional antennas.

IEEE 802.11ad [12] is one of the major existing RATs operating on the $60 \mathrm{GHz}$ unlicensed spectrum. Channel access in IEEE 802.11ad is based on an enhanced distributed channel access, where the physical carrier sensing on the PHY layer uses CCA and virtual carrier sensing on the medium access control (MAC) layer uses network allocation vector. The channel is considered as busy if either the physical or the virtual carrier sensing indicates it as busy. A unified and flexible beamforming scheme is also defined to support transmitter and receiver beam steering. Beamforming is mandatory in IEEE 802.11ad for both transmitter and receiver and involves sector-level sweep, beam refinement protocol, and beam tracking.

IEEE 802.11ay, expected to be released in 2017, also operates at $60 \mathrm{GHz}$ as an improvement of IEEE 802.11ad and targets larger transmission rates and extended transmission distances. The likely features in IEEE 802.11ay may include channel bonding, multi-user multiple-inputmultiple-output techniques, and directional LBT. For example, in multi-beam operation system, sensing along particular directions is considered to be beneficial to improve the channel access efficiency by alleviating the false alarm issue using omni-directional sensing. These design aspects can also be taken into consideration in the design of $5 \mathrm{G} N R$ unlicensed spectrum operation at $60 \mathrm{GHz}$ at least for ensuring fair co-existence with IEEE 802.11ad and IEEE 802.11ay.

\section{B) The $3.5 \mathrm{GHz}$ shared spectrum}

The $3.5 \mathrm{GHz}$ spectrum refers to the commercial shared use of a $150 \mathrm{MHz}$ bandwidth from 3.55 to $3.7 \mathrm{GHz}$, where licensed and unlicensed use of a variety of services is allowed. In 2015, the FCC in the USA released new rules that create a three-tiered commercial radio service, called Citizens Broadband Radio Service [13], that enables flexible spectrum sharing on the $3.5 \mathrm{GHz}$ band. The highest tier consists of the incumbent federal users and fixed satellite service operators and their interference protection from lower tiers is guaranteed. The second tier is Priority Access (PA) licensees that are authorized to utilize $10 \mathrm{MHz}$ channels in the $3.55-3.65 \mathrm{GHz}$ range. PA licensees can aggregate up to four channels and have to provide interference protection to the highest tier. The last tier is General Authorized Access (GAA) operators that are allowed to access up to $80 \mathrm{MHz}$ bandwidth that is not assigned to the highest tier or the second tier. GAA operators may not receive any interference protection from higher tiers.
Spectrum Access System (SAS) has been employed by FCC to manage the flexible spectrum sharing among the three tiers. The SAS coordinates the spectrum assignment based on reports from the Environmental Sensing Capability sensors regarding the presence or activity of incumbents and interference/congestion reports from PA/GAA users. In this manner, spectrum sharing is managed by a central coordinator and no LBT is mandatory on the $3.5 \mathrm{GHz}$ band.

\section{C) The 5 G NR unified framework for licensed and unlicensed spectrum}

Due to requirements for backward compatibility, LAA/ eLAA operates with $1 \mathrm{~ms}$ scheduling granularity, $4 \mathrm{~ms}$ latency between DL data transmission and associated HARQ-ACK feedback, 4 ms latency between uplink (UL) scheduling grant and associated UL data transmission, and with a maximum of $20 \mathrm{MHz}$ bandwidth per carrier. Those restrictions lead to suboptimal performance on unlicensed spectrum considering regulatory requirements, such as for MCOT and LBT, and co-existence with other RATs. For example, the $1 \mathrm{~ms}$ scheduling granularity does not allow for immediate data transmission after an LBT success, the $4 \mathrm{~ms}$ latency for HARQ-ACK feedback transmission or for UL data transmission can result to blocked transmissions due to MCOT limitations and/or LBT failures, and a maximum carrier bandwidth of $20 \mathrm{MHz}$ requires increased DL/UL control overhead.

The 5 G NR aims to address LAA/eLAA operational shortcomings by a unified framework for licensed and unlicensed spectrum where functionalities for operation on unlicensed spectrum are a subset of the overall $5 \mathrm{G}$ NR ones. First, $5 \mathrm{G}$ NR aims to increase a scheduling granularity to practically an arbitrary number of OFDM symbols and also support OFDM symbol durations that are several times smaller than the one in LAA/eLAA, for example, about $17.8 \mu \mathrm{s}$ instead of $71.4 \mu \mathrm{s}$ using a $60 \mathrm{KHz}$ subcarrier spacing instead of a $15 \mathrm{KHz}$ one. Second, $5 \mathrm{G}$ NR aims to achieve a much reduced latency between DL data transmission and associated HARQ-ACK feedback by utilizing a short OFDM symbol duration, such as $17.8 \mu \mathrm{s}$, and enabling variable HARQ-ACK timing. For example, HARQ-ACK associated with a number of DL data transmissions can be jointly provided and a turnaround time between a last DL data transmission and an associated HARQ-ACK feedback can be a short OFDM symbol and not require additional LBT. Third, 5G NR aims to practically eliminate a latency between UL scheduling grant and associated UL data transmission by supporting UL data transmissions autonomously from UEs

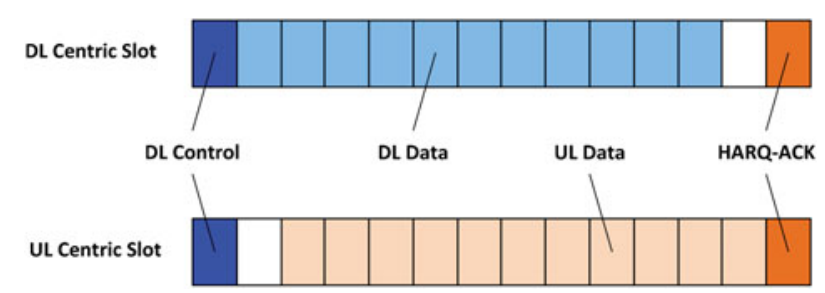

Fig. 6. Additional DL-centric and UL-centric slot structures in $5 \mathrm{G}$ NR. 
without respective UL scheduling grants. Finally, $5 \mathrm{G}$ NR aims to support a maximum carrier bandwidth in the order of $80-100 \mathrm{MHz}$, at least for higher carrier frequency bands while maintaining the $20 \mathrm{MHz}$ bandwidth granularity at the ${ }_{5} \mathrm{GHz}$ band for co-existence with Wi-Fi and LAA/eLAA. Figure 6 illustrates slot structures, additional to the typical DL-only or UL-only ones, considered in $5 \mathrm{G}$ NR to provide HARQ-ACK feedback in a same slot as for UL data transmission and, especially, as for DL data transmission.

\section{CONCLUSION}

LTE evolution has taken a big step forward in supporting joint radio access in both licensed and unlicensed bands with LAA, eLAA, and LTE-U technologies. It is also vital for the next-generation wireless communications standards such as $5 \mathrm{G}$ NR to efficiently support operations in various spectrum licensing regimes. Unlike LAA/eLAA where the system design had to maintain backward compatibility with LTE to a large extent, $5 \mathrm{G}$ NR represents a unique opportunity for designing a unifying framework for licensed, unlicensed, and shared bands from the beginning without the burden of any backward compatibility other than for co-existence with other RATs. While some design principles are still expected to be inherited from LAA and eLAA, particularly for the unlicensed $5 \mathrm{GHz}$ band for co-existence reasons, $5 \mathrm{G}$ NR design will be required to support operations in new spectrums with greater variations in regulatory and co-existence requirements, and channel characteristics among the different bands. The $5 \mathrm{G}$ NR is expected to consider/support new techniques, including but not limited to, enhanced LBT operations taking into account the signal directivity in unlicensed $60 \mathrm{GHz}$ and co-existence with IEEE 802.11ad/ay, operations with SAS and other users with different access priorities in shared bands such as the 3.5 $\mathrm{GHz}$ band in the USA, a unified flexible and dynamic slot structures, in conjunction with variable scheduling control signaling and HARQ-ACK timings with significantly reduced latency to enable better co-existence with other RATs.

\section{REFERENCES}

[1] RP-141664: Study on Licensed-Assisted Access Using LTE, 3 GPP TSG RAN Meeting \#65, September 2014.

[2] RP-151045: New Work Item on Licensed-Assisted Access to Unlicensed Spectrum.

[3] FCC Part 15 ruling: http://www.ecfr.gov/cgi-bin/text-idx?SID=3C5 e2d1533490603eo131fcdco4103od\&node $=$ pt 47.1.15\&rgn $=$ div 5

[4] ETSI EN 301 893: Harmonized European Standard, Broadband Radio Access Networks (BRAN); $5 \mathrm{GHz}$ high performance RLAN.

[5] LTE-U Forum: http://www.lteuforum.org/index.html

[6] RP-16o881: Revision of Work Item on enhanced LAA for LTE, ${ }_{3} \mathrm{GPP}$ TSG RAN Meeting \#72, June 2016.

[7] TS 36.211 V14.1.0 (2016-12): E-UTRA Physical channels and modulation (Release 14).

[8] TS 36.212 V14.1.o (2016-12): E-UTRA Multiplexing and channel coding (Release 14).
[9] TS 36.213 V14.1.o (2016-12): E-UTRA Physical layer procedures (Release 14).

[10] TR 36.889: Study on Licensed-Assisted Access to Unlicensed Spectrum

[11] RP-161596: Revision of SI: Study on New Radio Access Technology, 3GPP TSG RAN Meeting \#73, September 2016.

[12] IEEE 802.11: Wireless LANs standards http://standards.ieee.org/ about/get/802/802.11.html

[13] FCC Rulemaking: 3.5 GHz Band/Citizens Broadband Radio Service https://www.fcc.gov/rulemaking/12-354

BOON LOONG NG received the Bachelor of Engineering (Electrical and Electronic) degree and the Ph.D. degree in Engineering from the University of Melbourne, Australia, in 2001 and in 2007, respectively. He is currently a Senior Staff Engineer with Samsung Research America Ü Standards \& Mobility Innovation (SMI) Lab in Dallas, Texas. He is also the group leader for New Communications Technology Group, where the R\&D focus is on system designs of next generation communication systems. He has contributed to 3 GPP standards in RAN working groups since LTE Release 8 , and holds numerous patents on LTE/LTE-A/LTE-A Pro.

ARIS PAPASAKELLARIOU (aris.papas@samsung.com) is a Sr. Director with Samsung Research America. He received his Ph.D. degree from Rice University in 1996. He has been working on $3 \mathrm{GPP}$ standardization since 2005.

HONGBO SI received the B.S. degree in Maths and Physics, and the M.S. degree in Electronic Engineering from Tsinghua University, Beijing, China, in 2007 and 2010, respectively. He received the Ph.D. degree in Electrical and Computer Engineering from The University of Texas at Austin in 2015. He is currently a Staff Standards Engineer with Samsung Research America - Standards \& Mobility Innovation (SMI) Lab in Dallas, Texas. His research interests include information and coding theory, wireless communication, and bioinformatics. He spent the fall of 2014 and spring of 2015 interning at Samsung Research America. Hongbo is a recipient of the master thesis award and outstanding graduates award from Tsinghua University in 2010.

JIANZHONG CHARLIE ZHANG is a VP and head of Standards and Mobility Innovation Lab with Samsung Research America, where he leads research, prototyping and standards for $5 \mathrm{G}$ cellular systems and future multimedia networks. He received his Ph.D. degree from University of Wisconsin, Madison. From August 2009 to August 2013, he served as the Vice Chairman of the 3GPP RAN1 working group and led development of LTE and LTE-Advanced technologies such as $3 \mathrm{D}$ channel modeling, UL-MIMO and CoMP, Carrier Aggregation for TD-LTE, etc. Before joining Samsung, he was with Motorola from 2006 to 2007 working on 3 GPP HSPA standards, and with Nokia Research Center from 2001 to 2006 working on IEEE 802.16e (WiMAX) standard and EDGE/CDMA receivers. Dr. Zhang is a Fellow of IEEE. 